\title{
A Bacillus-based Coxsackie virus A16 mucosal vaccine induces strong neutralizing antibody responses
}

\author{
YIN-GUANG CAO ${ }^{1,2}, Y U H A O^{I}$, LEXIN WANG ${ }^{3,4}$ \\ ${ }^{1}$ Clinical Laboratory, Liaocheng People's Hospital of Taishan Medical University, Shandong, China \\ ${ }^{2}$ Shandong University School of Medicine, Shandong, China \\ ${ }^{3}$ School of Biomedical Sciences, Charles Sturt University, Wagga Wagga, NSW, Australia \\ ${ }^{4}$ Department of Cardiology, Liaocheng People's Hospital of Taishan Medical University, Shandong, China
}

\begin{abstract}
The purpose of this study was to construct a Coxsackie virus A16 (CA16) mucosal vaccine and evaluate its ability to induce immune response. VP1 gene of CA16 was inserted into the genome of Bacillus subtilis via recombination and displayed on the surface of the spores. This Bacillus-based vaccine was used for intranasal immunization of mice and the serum antibody titer was determined by enzyme-linked immunosorbent assay (ELISA). Neutralization activity of the serum from immunized mice was analyzed by an in vitro neutralizing test. VP1 gene was successfully integrated into the genome of Bacillus subtilis and was expressed on the surface of Bacillus spores. Intranasal immunization of mice with this vaccine induced a higher level of VPI specific IgA and IgG than in mice of the control group $(p<0.05)$. The neutralizing antibody titer in the spore immunization group was $1: 169$, which was higher than that in the control group $(p<0.05)$. We concluded that vaccine prepared by displaying CA16 VP1 protein on the surface of Bacillus subtilis spores can stimulate mice to produce protective neutralizing antibodies, which provides foundations for the development of CA16 mucosal vaccine.
\end{abstract}

Key words: Coxsackie virus A16, VP1, mucosa vaccine, Bacillus subtilis, genes, hand, foot and mouth disease.

(Centr Eur J Immunol 2019; 44 (1): 1-6)

\section{Introduction}

Hand, foot and mouth disease (HFMD) is a major threat to the health of infants [1-5]. In 2008, HFMD was listed as a class $\mathrm{C}$ infectious disease (which is mandatory to be reported) in the "People's Republic of China Disease Prevention Act". The pathogens that cause HFMD are mainly enterovirus 71 (EV71) and Coxsackie virus type A16 (CA16) [6-8]. CA16 also causes myocarditis, cardiomyopathy, pericarditis and other serious diseases, which accounts for more than $31.9 \%$ of the total number of infections [9]. CA16 was first isolated in Canada in 1958. CA16 was classified as a small RNA virus family (Picornaradae) and enterovirus genus (Enterovirus). The structural proteins of CA16 include VP1, VP2, VP3, and VP4, of which VP1, VP2, and VP3 were present on the viral surface, while VP4 is located near RNA. Although extensive studies on CA16 have been reported, effective therapeutic drugs and vaccines are still not available. Thus, it is difficult to prevent and treat CA16 infections by vaccines and drugs that are developed by conventional methods. In this study, we fused VP1 gene with CotB and introduced this recombinant gene into the genome of Bacillus subtilis. VP1 was displayed on the surface of Bacillus spores. This Bacillus-based mucosal vaccine could induce strong neutralizing antibody response in mice and thus could potentially be used to protect from CA16 infection.

\section{Material and methods}

This study was approved by the institutional review board of Liaocheng People's Hospital.

Plasmid pDG1662 and Bacillus subtilis 1A771 were purchased from Bacillus Genetic Stock Center (Columbus, USA). CA16 was isolated from the stool specimens of HFMD patients admitted to Liaocheng People's Hospital after obtaining written consent. BALB/c mice were purchased from the Experimental Animal Center of Shandong University (Jinan, China). Cloning vector pMD18-T and pfu DNA polymerase were purchased from TaKaRa Biotechnology (Dalian, China). The RNA extraction kit, DNA gel extraction kit and plasmid extraction kit were purchased from Tiangen Technology (Beijing, China). The restriction enzyme and reverse transcriptase kit were

Correspondence: Lexin Wang, MD, PhD, Department of Cardiology, Liaocheng People's Hospital, Liaocheng, Shandong Province,

China, 252000, e-mail: lwang@csu.edu.au

Submitted: 4.09.2016; Accepted: 5.02.2017 


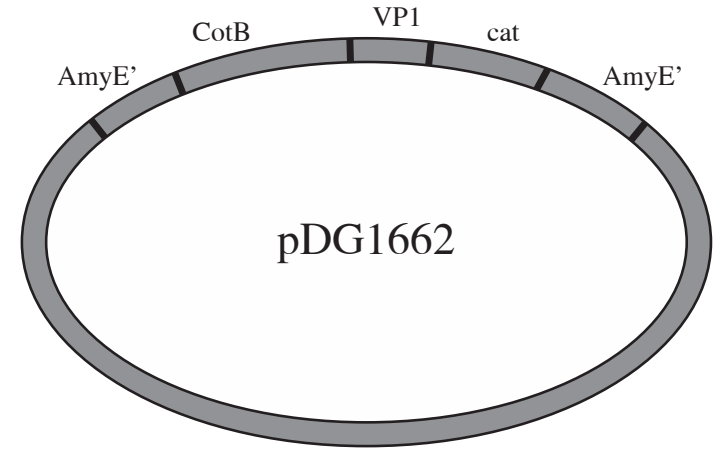

Fig. 1. Schematic representation of the fusion gene in pDG1662

purchased from Thermo (Waltham, MA USA). HRP conjugated goat anti-mouse IgG was purchased from Beijing Biosynthesis Biotechnology (Beijing, China). HRP conjugated goat anti-mouse IgA was purchased from Abcam (Cambridge, UK). FITC-conjugated goat anti-mouse IgG was purchased from Wuhan Boster Biological Engineering (Wuhan, China). Complete Freund's adjuvant and incomplete Freund's adjuvant were purchased from Sigma (Shanghai, China). Chemical reagents were purchased from Sinopharm Chemical Reagent (Shanghai, China).

\section{PCR primers}

CotB and VP1 genes were amplified by the following primers:

CotB 1: 5' - GAA T TCGAA TCCGAGTTTCG-

CAAGTCCT-3';

CotB2: 5' - AAGCTTGATGATTGATCATCT-

GAAGAT-3' (expected size: 1305 bp);

VP1.1: 5'-AAGCTTGGGGACCCCATTGCAGATAT-

GAT-3';

VP1.2: 5'-GGATCCCAGCGTTGTTATCTTGTCTC-

TACT-3' (expected size: 903 bp).

Primers were synthesized by Shanghai Boya Bio Co (China). RNA Extraction Kit was used to isolate CA16 genome and Oligo (dT) was used as the primer to obtain cDNA by the reverse transcription. cDNA and Bacillus 1A771 DNA were used as templates to amplify the VP1 and CotB genes, respectively. The amplified polymerase chain reaction (PCR) products were ligated into pMD18-T vector (resulting in the plasmids pMD18-VP1 and pMD18CotB, respectively) and confirmed by DNA sequencing.

\section{Construction of recombinant plasmids}

Plasmids pMD18-VP1 and pMD18-CotB were double digested by restriction enzyme pair of EcoRI/HindIII and HindIII/BamHI, respectively. The digested fragments were ligated into pDG1662 that was digested with the EcoRI and BamHI (Fig. 1). The resulting plasmids containing VP1 and CotB fusion gene were verified by PCR using
CotB1-CotB2, VP1.1-VP1.2 and CotB1-VP1.2 primers and restriction digestion using EcoRI/HindIII, HindIII/ BamHI and EcoR/BamHI.

\section{Transformation and screening}

A loop of 1 A771 spores were inoculated into $5 \mathrm{ml} \mathrm{me-}$ dium and cultured overnight. The overnight culture was inoculated into $80 \mathrm{ml}$ growth medium and incubated for 3 hours with shaking. The culture was incubated on ice for $10 \mathrm{~min}$ and centrifuged $\left(4^{\circ} \mathrm{C}, 5000 \times \mathrm{g}\right)$ to collect the bacterial pellet. Competent cells were prepared by washing the Bacillus 3 times using cold electroporation buffer. Plasmid pDG1662-CotB-VP1 was linearized by restriction enzyme NdeI and incubated with the competent cells for $1.5 \mathrm{~min}$. Following electroporation $(1800 \mathrm{~V}), 1 \mathrm{ml}$ recovering medium was added to the cuvette and cultured for 3 hours. The culture was spread on LB plate with chloramphenicol. Colonies that are resistant to chloramphenicol, but sensitive to erythromycin, were the correct recombinant strain.

\section{Verification of the recombinant strain by PCR}

Colonies that were resistant to chloramphenicol, but sensitive to erythromycin, were cultured in $10 \mathrm{ml} \mathrm{LB}$ containing chloramphenicol overnight. Genomic DNA was extracted by phenol chloroform. VP1 primers were used to verify if the recombinant strains were correctly constructed.

\section{Verification of the recombinant strain \\ by Western blot}

Chloramphenicol resistant strain was cultured in LB at $37^{\circ} \mathrm{C}$ with shaking for 24 hours followed by static standing at room temperature for 48 hours to generate sufficient spores. Cells were collected by centrifugation $(10000 \times \mathrm{g})$ and treated with lysozyme $(20 \mathrm{mmol} / \mathrm{l}$ Tris- $\mathrm{HCl}$ buffer solution, $\mathrm{pH} 8.0$, lysozyme $1 \mathrm{~g} / \mathrm{l}$ ) at $37^{\circ} \mathrm{C}$ for 1 hour. Spores were collected by centrifugation. Shelling buffer $(10 \mathrm{ml})$ was used to resuspended spores. Spores were incubated at $70^{\circ} \mathrm{C}$ with $250 \mathrm{rpm}$ shaking for 1 hour. Following centrifugation at $14000 \times \mathrm{g}$, the supernatant contains capsid protein. Wild-type strain was prepared similarly. Capsid protein and $2 \times$ sample buffer was mixed and boiled for $3 \mathrm{~min}$. After SDS-PAGE and transferring to nitrocellulose membrane, western blot was performed using polyclonal anti-CA16 as the primary antibody and HRP conjugated goat anti-mouse IgG as the secondary antibody.

\section{Characterization of the recombination strain by fluorescent microscopy}

Spores were washed with phosphate-buffered saline (PBS) for 3 times and incubated with CA16 polyclonal antibody overnight. Following 3 times of washing with PBST, the spores were resuspended and incubated with FITC-conjugated goat anti-mice IgG for 1 hour at $37^{\circ} \mathrm{C}$ with slight 
shaking. Subsequently, the spores were washed and placed on the glass slide for fluorescent microscopic analysis.

\section{Animal immunization}

A total of $26 \mathrm{BALB} / \mathrm{c}$ mice were randomly divided into experimental and control groups $(n=10$ per group) and the remaining 6 mice were used as a negative group. The experimental, control and negative groups were inoculated with the recombinant Bacillus, VP1 protein and saline solution, respectively, via intranasal immunization twice a week for a total of 6 weeks. At week 7, blood sample was collected from ophthalmic artery plexus and the serum was stored at $-80^{\circ} \mathrm{C}$ for later use.

\section{Measurement of antibody titer by ELISA}

The 96-well plate was coated with inactivated CA16 virus and blocked by $5 \%$ non-fat milk. The serum was 2 -fold diluted with PBS and added into the each well (100 $\mu \mathrm{l} /$ well). After incubation at $37^{\circ} \mathrm{C}$ for 1 hour, HRP-conjugated goat anti-mouse immunoglobulin (Ig) $\mathrm{G}$ and IgA was added. Following additional 1 hour's incubation at $37^{\circ} \mathrm{C}$, TMB substrate was added. A450 was measured after the reaction was terminated by sulfuric acid (2M). SPSS13.0 was used to analyze the antibody titer in the serum of mice.

\section{Neutralization test}

Neutralization test was performed as described previously [10-12]. Briefly, mouse serum was diluted at $1: 10$ and inactivated at $56^{\circ} \mathrm{C}$ for 30 minutes. Inactivated serum was serially diluted at $1: 10$. Serum at each dilution was added with an equal volume of virus $\left(400\right.$ TCID $\left._{50}\right)$ and incubated for 1 hour at $37^{\circ} \mathrm{C}$. Each dilution was then inoculated onto 96 -well plate $(50 \mu \mathrm{l} /$ well $)$. Each well was also added with $50 \mu \mathrm{l}\left(1 \times 10^{4}\right) \mathrm{RD}$ cells. Following culture at $37^{\circ} \mathrm{C}$ and $5 \% \mathrm{CO}_{2}$ condition, pathological alterations of the cells were determined. The virus control, and normal serum control and normal cell control were included. The highest dilution without pathological alteration was considered as the neutralizing antibody titers. SPSS13.0 was used for data analysis.

\section{Results}

\section{Amplification of the VP1 and CotB genes}

PCR results showed that CotB and VP1 genes were successfully amplified with an expected size of 1305 and 904 bp, respectively (Fig. 2). Sequence analysis showed that CotB and VP1 genes were inserted into pMD18-T vector, resulting in the plasmids pMD18-VP1 and pMD18CotB, respectively.

\section{Construction of the recombinant plasmid}

Plasmids pMD18-VP1 and pMD18-CotB were double digested with the restriction enzyme pair of EcoR/HindIII

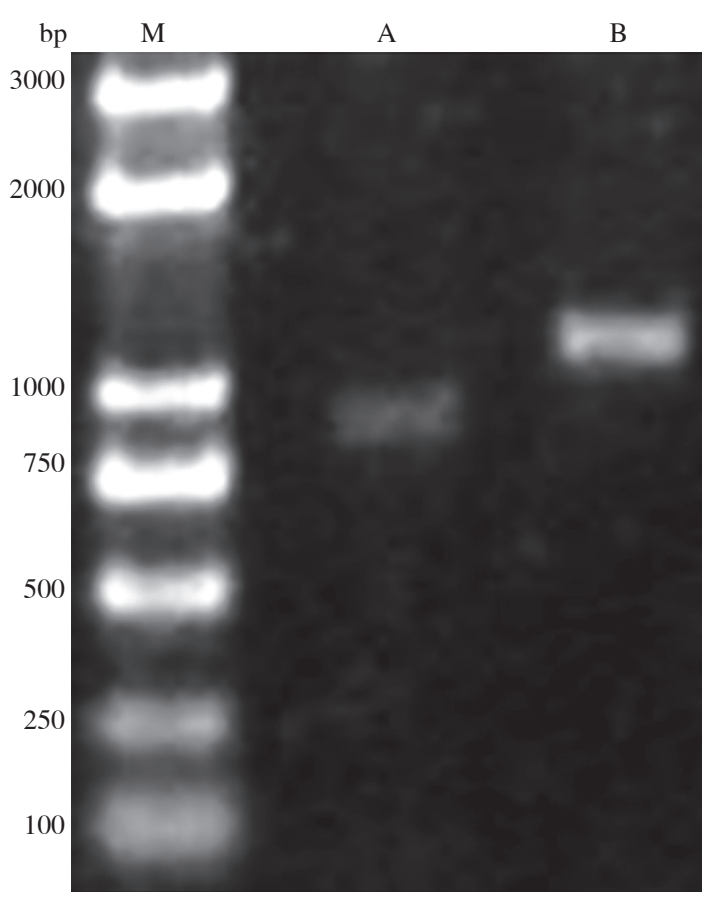

Fig. 2. Agarose gel electrophoresis of PCR product. $\mathrm{M}$ - Marker, A - PCR product of VP1, B - PCR product of CotB

and HindIII/BamHI, respectively. The fragments (CotB and VP1) excised from the two plasmids were inserted into pDG1662, resulting in the recombinant plasmid pDG1662CotB-VP1. Restriction digestion of pDG1662-CotB-VP1 with EcoR/HindIII, HindIII/BamHI and EcoR/BamHI resulted in the expected bands of 1305bp, 904bp and 2209bp, respectively (Fig. 3), which indicated that CotB and VP1 fusion was constructed into pDG1662 plasmid.

\section{Characterization of the recombinant strain by PCR}

PCR using genomic DNA extracted from the recombinant strain as template and VP1.1-VP1.2 as primers resulted in an expected size of $904 \mathrm{bp}$ band, while the control did not have positive band (Fig. 4), which indicated that VP1 was successfully inserted into the genome of Bacillus subtilis.

\section{Characterization of the recombinant strain by Western blot}

Proteins were extracted from wild-type and recombinant Bacillus subtilis spores and resolved by SDS-PAGE. Western blot was performed using VP1 polyclonal antibody as the primary antibody and HRP-conjugated goat anti-mouse IgG as the secondary antibody. The results showed a positive band in the recombinant strain, but not in the wild-type strain (Fig. 5), which indicated that VP1 was expressed in the spores of the recombinant strain. 


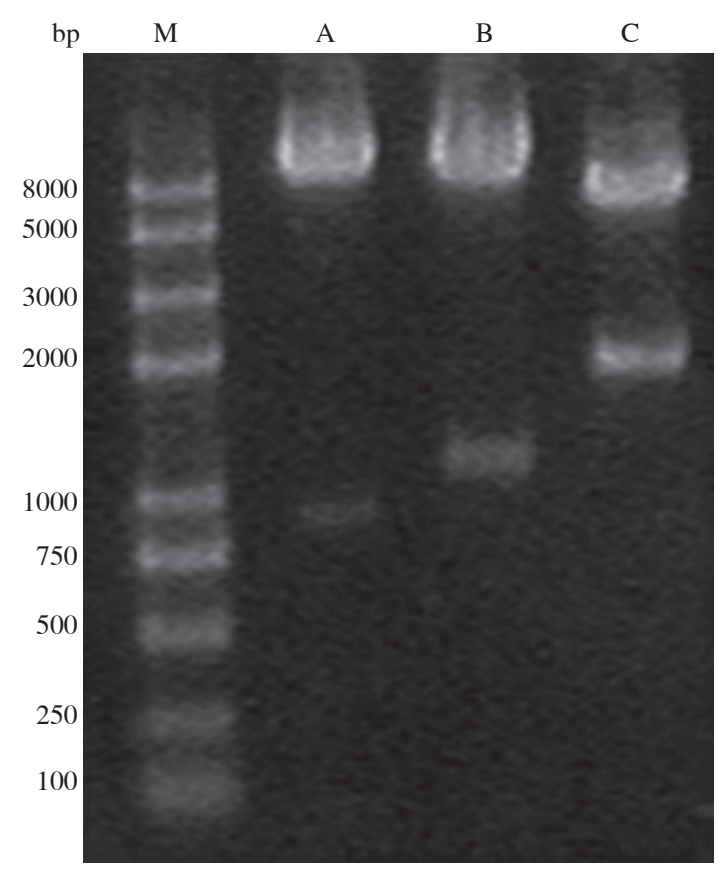

Fig. 3. Agarose gel electrophoresis of the products that are digested by double restriction enzymes. $\mathrm{M}-$ marker, $\mathrm{A}$ - product of pDG1662-cotB-VP1 digested by HindIII/ BamHI, B - product of pDG1662-cotB-VP1 digested by EcoRI/HindIII, C - product of pDG1662-cotB-VP1 digested by EcoRI/BamHI

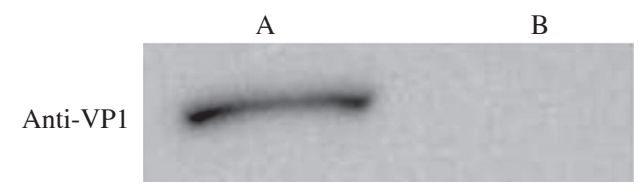

Fig. 5. Western blot analysis of spore coat proteins. A - recombinant strain, B - wild-type strain

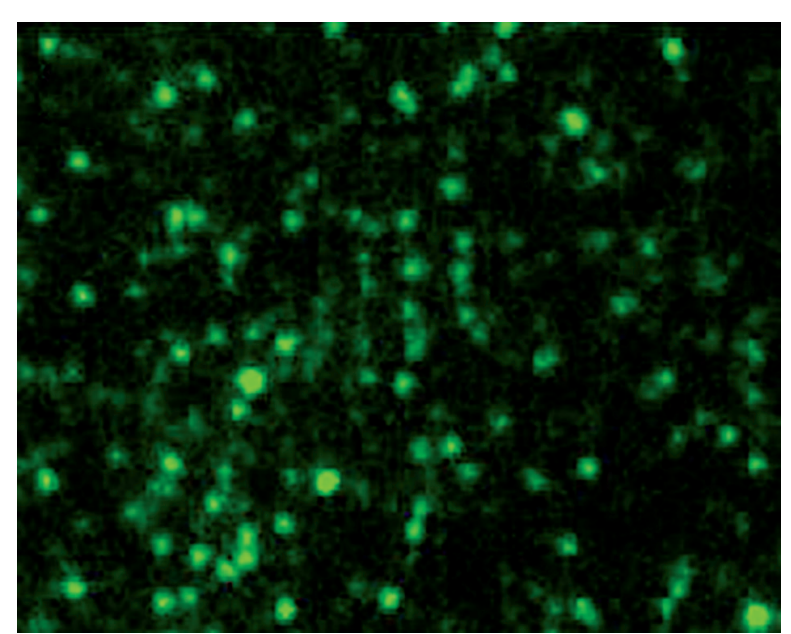

Fig. 6. Immunofluorescence microscopy analysis. Phase of fluorescence microscope of recombination strain

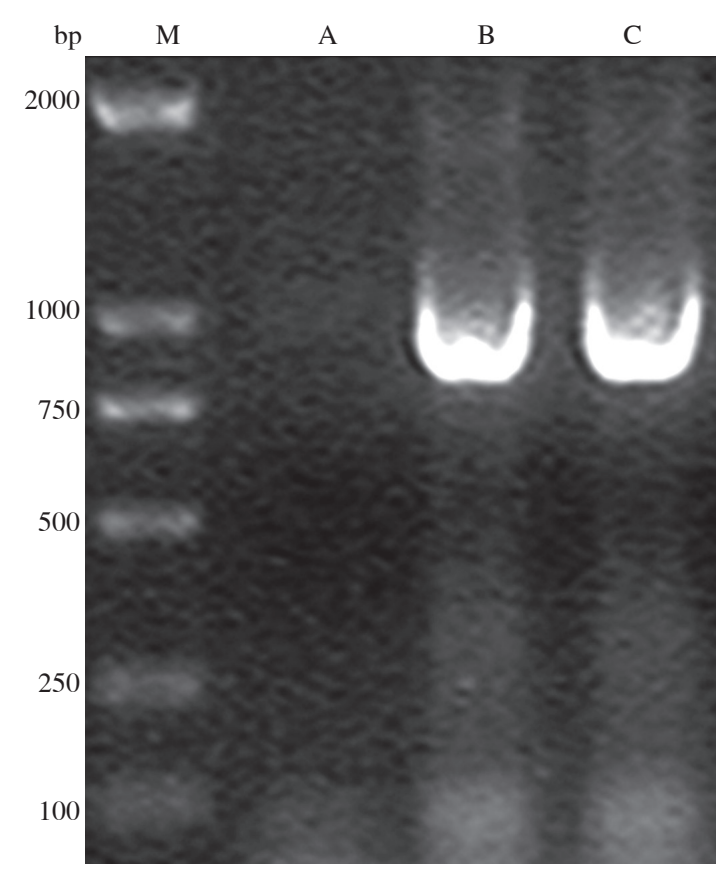

Fig. 4. Agarose gel electrophoresis of PCR product amplified using Bacillus subtilis genome as template. $\mathrm{M}$ - marker, A - PCR product of wild-type strain, B, C - PCR product of recombinant strain

\section{Characterization of the recombinant strain by immunofluorescence microscopy}

The spores of the recombinant and wild-type strains were incubated with VP1 polyclonal antibody and FITC-conjugated goat anti-mouse IgG. Fluorescence microscopy showed that the recombinant strain exhibited green fluorescence (Fig. 6), while the wild-type strain did not exhibit green fluorescence (Fig. 6). These results indicated that VP1 was displayed on the surface of spores and had strong immunogenicity.

\section{Antibody titers measured by ELISA}

Mice were immunized with the recombinant strain and the antibody including $\operatorname{IgG}$ and $\operatorname{IgA}$ in the serum was measured by ELISA using CA16 as antigen. The results showed that CA16 specific antibody was induced in mice. The highest and lowest titer of IgA was $1: 1280$ and $1: 320$ (geometric mean titer was $1: 597$ ), respectively. The highest and lowest titer of $\operatorname{IgG}$ was $1: 5120$ and $1: 640$ (geometric mean titer was $1: 2087$ ), respectively (Fig. 7). The antibody titers in the mice immunized with the recombinant strain was significantly higher than those immunized with VP1, which suggested that intranasal immunization induced specific humoral immune response. The spores can significantly increase the antibody level and can function as an excellent mucosal adjuvant. 


\section{Cell neutralizing assay}

Serially diluted serum from each group of mice was used for neutralizing assay. The results showed that the highest and lowest titer of the antibody from the recombinant strain group was $1: 512$ and $1: 64$ (geometric mean titer was $1: 186$ ), respectively (Fig. 8). The titer in the recombinant strain group was significantly higher than that in the VP1 control group (Fig. 8), which indicated that immunization with spore not only increases the ELISA titer, but also enhances the neutralizing antibody titers.

\section{Discussion}

CA16 is one of the main pathogens causing HFMD in mainland China [13-15]. Extensive studies on CA16 vaccine have been reported. Virus-like particle and inactivated virus can protect suckling mice from lethal doses of virus attacks $[16,17]$. The subunit vaccines study mainly focused on VP1 capsid protein. It is well known that CA16 capsid protein is composed of VP1-4, of which protein VP1 is the major capsid protein and has a specific neutralizing epitope. Immunization of animals with VP1 can induce strong humoral and cellular immune response [18]. Thus, VP1 is the best choice for preparing CA16 subunit vaccine. However, whole virus or subunit vaccine researches are still in the experimental stage and alternative approaches are needed to develop an ideal CA16 vaccine. $\operatorname{IgA}$ is one of the important immunoglobulins. $\operatorname{IgA}$ is particularly important in resistance to pathogenic microorganisms, especially those enteric viruses that cause infections via the mucosal route. $\operatorname{IgA}$ is thought to protect the host against pathogens that infect mucosal surfaces and those that cause systemic disease after entry via a mucosal surface [19]. Therefore, induction of high levels of IgA and IgG antibodies through the mucosal immunization route may provide new strategies to prevent and control CA16 infection. It has been reported that EV71 vaccine prepared by displaying VP1 protein on baculovirus can effectively induce immune response in mice [20]. Bacterial surface display technology, which is similar to the baculovirus display, also received widespread attention in the field of vaccine. Extensive studies using spore as the vaccine vector have been reported [21-23]. The spore vector is safe, stable, easy to prepare, and becomes a potentially effective tool for vector vaccine. Many studies have shown that mucosal subunit vaccine VA16 VP1 using spore as a vector is feasible.

In this study, we amplified VP1 gene from CA16 and inserted VP1 in fusion with CotB gene from Bacillus subtilis into pDG1662 plasmid. VP1 was inserted into the genome of Bacillus via recombination. Erythromycin sensitive and chloramphenicol resistant clones were selected. During the process of screening, we found that not all chloramphenicol resistant clones were sensitive to erythromycin, which suggested that erythromycin resistant gene

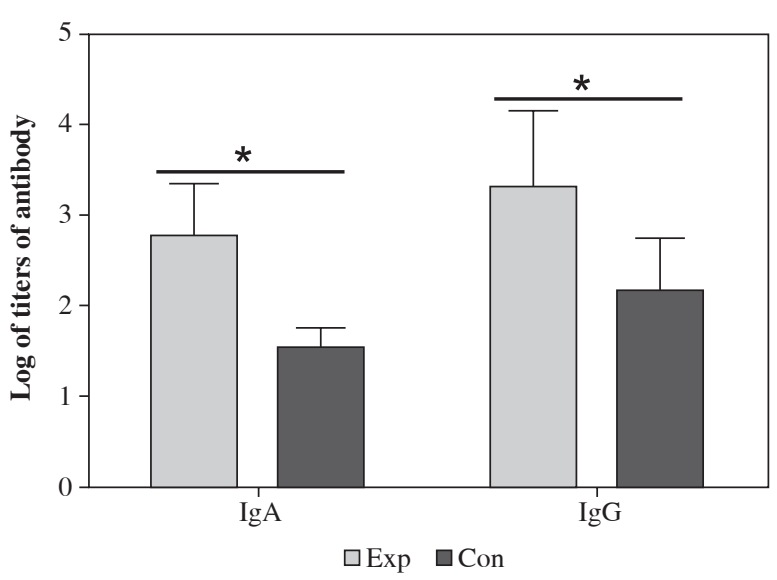

Fig. 7. The titers of $\operatorname{IgA}$ and $\operatorname{IgG}$ in serum samples as detected by ELISA

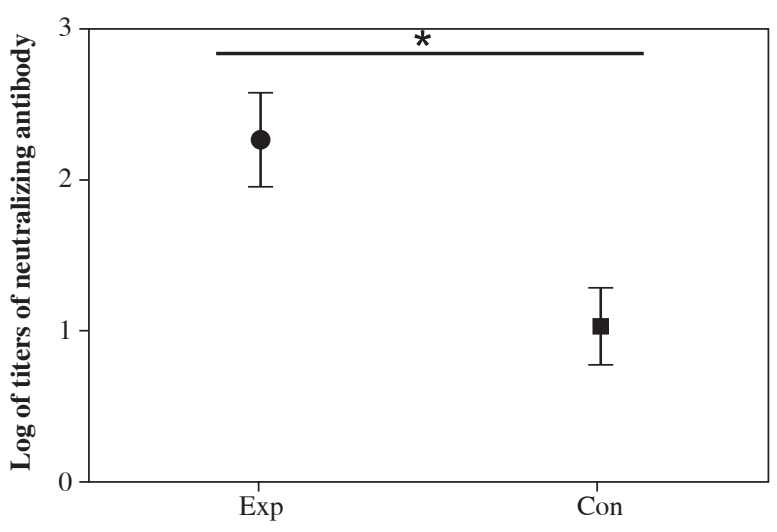

Fig. 8. Neutralizing antibody titers of mice serum samples

was not excised during the recombination. It also indicated that there were different ways of integration of pDG1662 into the genome. PCR, Western blot and immunofluorescence microscopy analysis demonstrated that VP1 gene was successfully integrated into the genome of Bacillus subtilis and was expressed on the surface of Bacillus spores. However, it is worth noting that the band in Western blot was weak, which indicated that VP1 expression was low, which is consistent with previous reports [24].

Intranasal immunization of mice with this vaccine induces a significantly higher level of VP1 specific IgA and IgG compared the non-immunized group, which suggested that mucosal immunization of VP1 using spore as adjuvant can enhance immunization efficacy and humoral immune response. More importantly, the neutralizing antibody titer in the spore immunization group was $1: 169$, which was significantly higher than that in the control group. These results indicated that mucosal vaccine using spore as a vector can increase not only the titer of the specific antibody, but also the titer of the neutralizing antibody. 
In summary, this study reported, for the first time, the CA16 VP1 subunit vaccine using spore as the expression system and adjuvant. This vaccine can effectively stimulate a specific neutralizing antibody via intranasal immunization and exhibit virus inhibitory activities. Our study provides new strategies to develop CA16 vaccine and sets the foundation for CA16 mucosal vaccine. This work represents an advance in biomedical science because it is introducing a new vaccine for the prevention of CA16 infection for the first time.

\section{Acknowledgements}

This study was supported by grants from the Natural Science Foundation of Shandong Province (ZR2009DQ025), China Postdoctoral Foundation (2014M561937), Medical and health science and technology development projects of Shandong Province (2014WS0299).

\section{The authors declare no conflict of interest.}

\section{References}

1. Kumar A, Shukla D, Kumar R, et al. (2012): Molecular epidemiological study of enteroviruses associated with encephalitis in children from India. J Clin Microbiol 50: 3509-3512.

2. Ni H, Yi B, Yin J, et al. (2012): Epidemiological and etiological characteristics of hand, foot, and mouth disease in Ningbo, China, 2008-2011. J Clin Virol 54: 342-348.

3. Zhu Q, Hao Y, Ma J, et al. (2011): Surveillance of hand, foot, and mouth disease in Mainland China (2008-2009). Biomed Environ Sci 24: 349-356.

4. Fan X, Jiang J, Liu Y, et al. (2013): Detection of human enterovirus 71 and Coxsackievirus A16 in an outbreak of hand, foot, and mouth disease in Henan Province, China in 2009. Virus Genes 46: 1-9.

5. Qiaoyun F, Xiongfei J, Lihuan L, Angao X (2013): Epidemiology and etiological characteristics of hand, foot and mouth disease in Huizhou City between 2008 and 2011. Arch Virol 158: 895-899.

6. Chen L, Mou X, Zhang Q, et al. (2012): Detection of human enterovirus 71 and coxsackievirus A16 in children with hand, foot and mouth disease in China. Mol Med Rep 5: 1001-1004.

7. Du J, Wang X, Hu Y, et al. (2014): Changing aetiology of hand, foot and mouth disease in Linyi, China, 2009-2011. Clin Microbiol Infect 20: O47-49.

8. Owatanapanich S, Wutthanarungsan R, Jaksupa W, Thisyakorn U (2016): Risk factors for severe enteroviral infections in children. J Med Assoc Thai 99: 322-330.

9. Xu M, Su L, Cao L, et al. (2015): Genotypes of the enterovirus causing hand foot and mouth disease in Shanghai, China, 2012-2013. PLoS One 10: e0138514.

10. Peng X, Fang X, Li J, et al. (2016): Enhancing immune responses of EV71 VP1 DNA vaccine by co-inoculating plasmid IL-12 or GM-CSF expressing vector in mice. Cell Mol Biol (Noisy-le-grand) 62: 35-41.

11. Yang C, Deng C, Wan J, et al. (2011): Neutralizing antibody response in patients with hand, food and mouth disease to enterovirus 71 and its clinical implications. Virol J 8: 306.
12. Wang X, Xing M, Zhang C, et al. (2014): Neutralizing ibody responses to enterovirus and adenovirus in healthy adults in China. Emerg Microbes Infect 3: e30.

13. Li W, Zhang X, Chen X, et al. (2015): Epidemiology of childhood enterovirus infections in Hangzhou, China. Virol J 12: 58.

14. Liu Y, Duan C, Zhang C, et al. (2015): Evaluation of a viral microarray based on simultaneous extraction and amplification of viral nucleotide acid for detecting human herpesviruses and enteroviruses. PLoS One 10: e0117626.

15. Tian H, Yang QZ, Liang J, et al. (2012): Clinical features and management outcomes of severe hand, foot and mouth disease. Med Princ Pract 21: 355-359.

16. Liu Q, Yan K, Feng Y, et al. (2012): A virus-like particle vaccine for coxsackievirus A16 potently elicits neutralizing antibodies that protect mice against lethal challenge. Vaccine 30: 6642-6648.

17. Cai Y, Liu Q, Huang X, et al. (2013): Active immunization with a Coxsackievirus A16 experimental inactivated vaccine induces neutralizing antibodies and protects mice against lethal infection. Vaccine 31: 2215-2221.

18. Shi J, Huang X, Liu Q, Huang Z (2013): Identification of conserved neutralizing linear epitopes within the VP1 protein of coxsackievirus A16. Vaccine 31: 2130-2136.

19. Lamm ME (1997): Interaction of antigens and antibodies at mucosal surfaces. Annu Rev Microbiol 51: 311-340.

20. van Ginkel FW, Nguyen HH, McGhee JR (2000): Vaccines for mucosal immunity to combat emerging infectious diseases. Emerg Infect Dis 6: 123-132.

21. Kang SM, Compans RW (2003): Enhancement of mucosal immunization with virus-like particles of simian immunodeficiency virus. J Virol 77: 3615-3623.

22. Ryan EJ, Daly LM, Mills KH (2001): Immunomodulators and delivery systems for vaccination by mucosal routes. Trends Biotechnol 19: 293-304.

23. Rosenthal KL, Gallichan WS (1997): Challenges for vaccination against sexually-transmitted diseases: induction and long-term maintenance of mucosal immune responses in the female genital tract. Semin Immunol 9: 303-314.

24. Duc le H, Hong HA, Uyen NQ, Cutting SM (2004): Intracellular fate and immunogenicity of B. subtilis spores. Vaccine 22: $1873-1885$. 